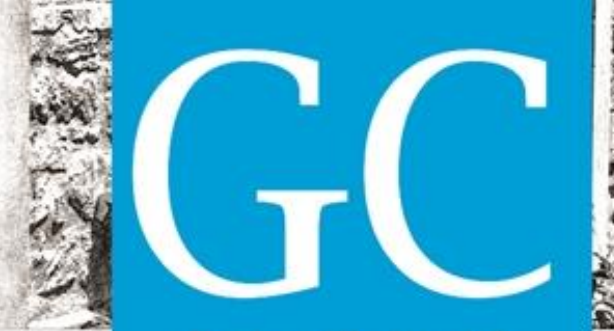

Revista Nacional de

Gerenciamento de Cidades

\title{
Avaliação de qualidade e vitalidade de espaços públicos de permanência: a Praça Luiz Zuiani, em Bauru-SP
}

Quality and vitality evaluation of public spaces of permanence: Luiz Zuiani Square, in Bauru-SP

Evaluación de calidad y vitalidad de espacios públicos de permanencia: Plaza Luiz Zuiani, en Bauru-SP

Eduarda de Mattos Previero Mestranda do Programa de Pós-Graduação em Arquitetura e Urbanismo, UNESP, Brasil. dudampreviero@gmail.com

Maria Solange Gurgel de Castro Fontes Professora Doutora, UNESP, Brasil. solange.fontes@unesp.br

Renata Cardoso Magagnin Professora Doutora, UNESP, Brasil. renata.magagnin@unesp.br 


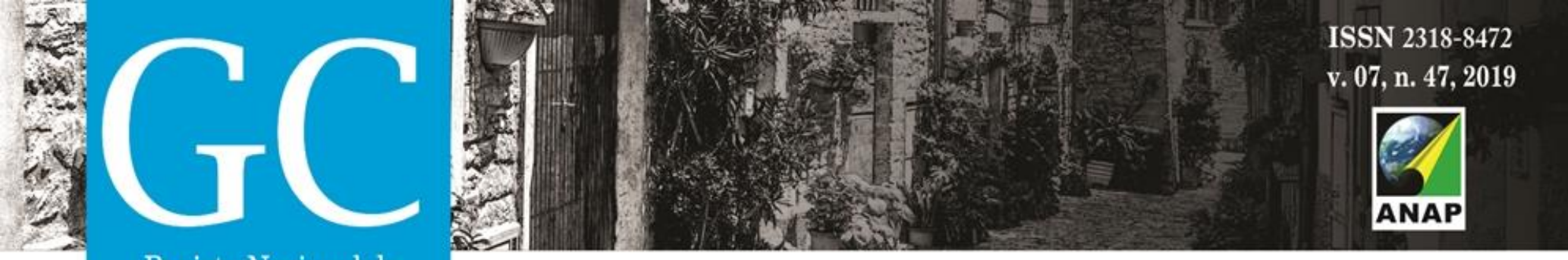

Revista Nacional de Gerenciamento de Cidades

\section{RESUMO}

As praças são locais de socialização e permanência. Elas devem conter qualidades que atraiam o usuário a permanecer nela e não apenas utilizar para passagem. Desta forma, o objetivo desse artigo é avaliar a qualidade de uma praça por meio de um estudo de caso na Praça Luiz Zuiani em Bauru e identificar seus pontos fortes e os que precisam de melhorias. Para isso é utilizado o método proposto pelo Project for Public Spaces (PPS), que divide as características necessárias em um espaço público em 4 categorias e as avalia por meio de perguntas. As questões são respondidas com "sim", "não" ou "parcial" e recebem uma nota ao final para que possa ser feita a classificação do espaço em: péssimo, ruim, regular, bom ou ótimo. Os resultados demonstraram que a praça é de ótima qualidade, pois é de fácil acesso, apresenta várias possibilidades de atividades, tem diversidade de usuários e é utilizada para socialização. Porém, ela possui pontos negativos, principalmente, atrelados a falta de manutenção do espaço, tanto nos equipamentos, caminhos e jardins como na limpeza do local. O estudo mostra que mesmo com problemas de manutenção a praça ainda é muito utilizada, pois tem uma variedade de usos que atrai os usuários ao local, é agradável e transmite segurança. O método utilizado foi de fácil aplicação e obteve resultados muito claros, por isso recomenda-se sua utilização para identificação de pontos que necessitam de melhorias em outros espaços públicos. PALAVRAS-CHAVE: Método de avaliação. Espaço público. Praça.

\section{ABSTRACT}

Squares are places of socialization and permanence. They must contain qualities that entice the user to stay in it and not just use it for passage. Therefore, the objective of this article is to evaluate a square quality through a case study in the Luiz Zuiani Square in Bauru and to identify its strengths and features that need improvement. To do that, is used the method proposed by the Project for Public Spaces (PPS), which divides the required features in a public space into 4 categories and evaluates them through questions. The questions are answered with "yes", "no" or "partial" and receive a note at the end so that the space can be classified as: terrible, bad, regular, good or great. The results showed that the square has great quality, because it is easy to access, presents several possibilities of activities, has a diversity of users and is used for socialization. However, it has negative points, mainly, due to lack of maintenance in the space, the equipment, paths and gardens as well as in the cleaning of the place. The study shows that even with maintenance problems the square is still widely used, because it has a variety of uses that attract users to the place, is pleasant and conveys safety. The method used was easy to apply and obtained very clear results, so its use is recommended for identification of points that need improvement in other public spaces.

KEYWORDS: Evaluation method. Public place. Square.

\section{RESUMEN}

Las plazas son sitios de socialización y permanencia. Ellas deben contener calidades que sean atractivas al usuario y le haga permanecer en ella y no solo la use como pasaje. De este modo, el objetivo de ese artículo es evaluar la calidad de una plaza a través de un estudio de caso en la Plaza Luiz Zuiani en Bauru e identificar sus puntos fuertes y los que necesitan ser mejorados. Para eso es utilizado el método propuesto por el Project for Public Spaces (PPS), que divide las características necesarias en un espacio público en 4 categorías y las evalúa por medio de preguntas. Las cuestiones son contestadas con "si", "no" o "parcial" y reciben una nota al final para se pueda hacer la clasificación del espacio en: pésimo, malo, regular, bueno u óptimo. Los resultados demostraron que la plaza es de óptima calidad, pues es de fácil acceso, tiene diversas posibilidades de actividades, posee diversidad de usuarios y es utilizada para socialización. Sin embargo, ella posee puntos negativos, principalmente, vinculados a falta de manutención del espacio, tanto en los equipamientos, caminos y jardines como en la limpieza del lugar. El estudio muestra que, aunque haya problemas de manutención, la plaza aún es muy utilizada, pues presenta una variedad de usos que atrae los usuarios al local, es agradable y trasmite seguridad. El método utilizado fue de fácil aplicación y obtuvo resultados muy claros, por eso su uso es recomendado para identificación de puntos que necesitan mejorías en otros espacios públicos.

PALABRAS-CLAVE: Método de evaluación. Espacio público. Plaza. 


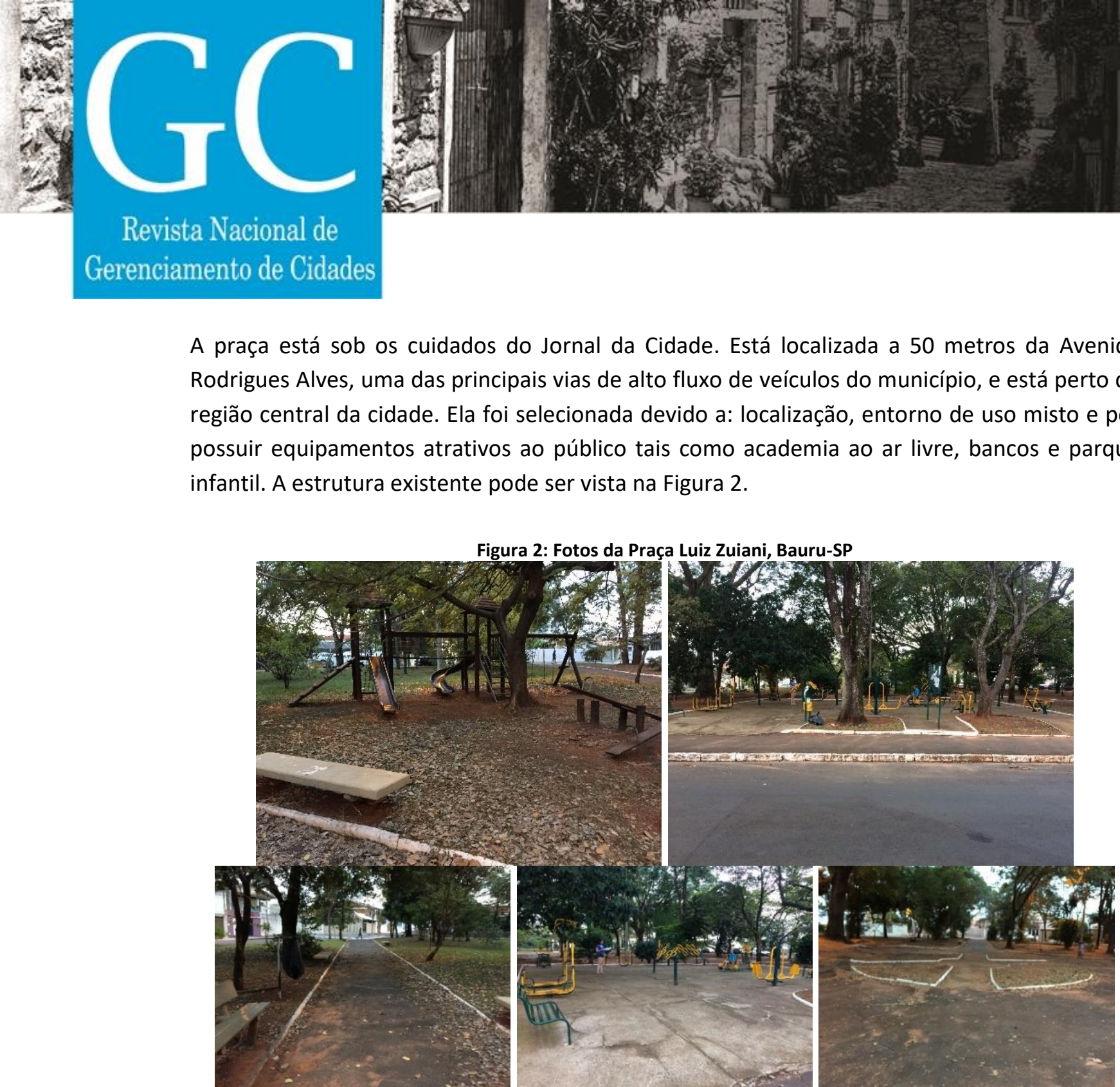

A praça está sob os cuidados do Jornal da Cidade. Está localizada a 50 metros da Avenida Rodrigues Alves, uma das principais vias de alto fluxo de veículos do município, e está perto da região central da cidade. Ela foi selecionada devido a: localização, entorno de uso misto e por possuir equipamentos atrativos ao público tais como academia ao ar livre, bancos e parque

Fonte: acervo das autoras, 2018.

\subsection{Escolha e aplicação do método de avaliação}

O método de avaliação selecionado é o elaborado pelo PPS, cuja denominação é "O que faz um ótimo lugar?” (PPS, 2018). Ele já foi abordado por diversos autores (FERNANDES, 2012; SAKIP, AKHIR, OMAR, 2014; KOSSAK-JAGOCZINSKA, 2015). O PPS divide espaços bem-sucedidos em 4 categorias: acessos e conexões, conforto e imagem, usos e atividades e sociabilidade. Cada categoria possui um sistema de perguntas que devem ser respondidas para avaliar o espaço.

As questões que devem ser respondidas para avaliar os acessos e conexões do espaço público são:

- Você pode ver o espaço à distância? Seu interior é visível do lado de fora?

- Existe uma boa conexão entre o espaço e os prédios adjacentes? ou é isolado e cercado por paredes vazias? Os ocupantes de edifícios vizinhos usam o espaço?

- As pessoas podem chegar caminhando com facilidade até o local? Elas têm que se apressar entre carros em movimento para chegar ao local ou é um percurso seguro?

- As calçadas levam de e para as áreas adjacentes? 


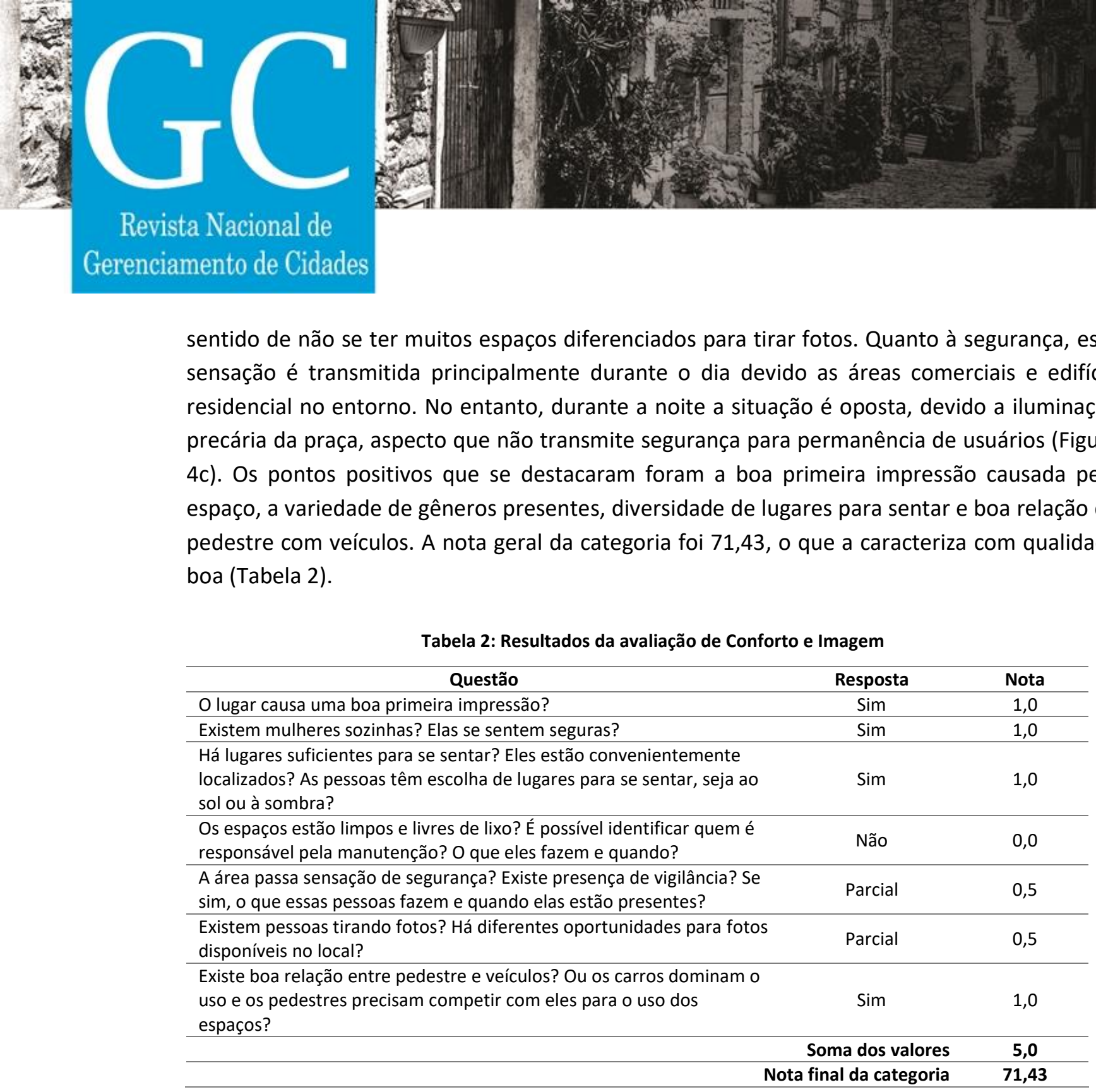

Fonte: elaborado pelos autores, 2019.

Figura 4: Fotos referentes a Conforto e Imagem: (a) presença de bancos; (b) lixeira precária; (c) praça a noite.

(a)

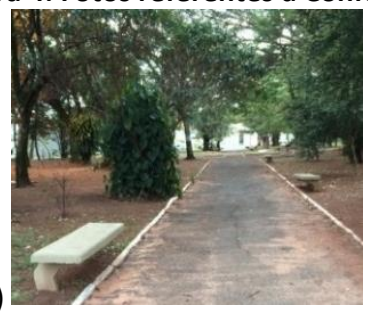

(b)

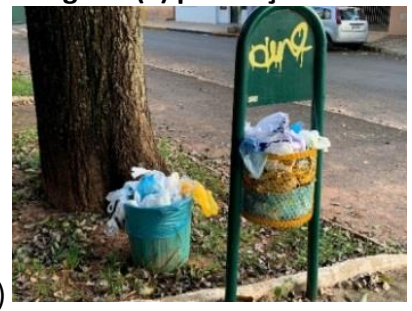

Fonte: acervo dos autores, 2018.

Quanto aos usos e atividades, apenas o quesito da presença de gerenciamento no local que prejudicou a pontuação. Isso ocorre porque apesar da placa de identificação do local dizer que a praça está aos cuidados do Jornal da Cidade, não é visível no local um bom gerenciamento. A situação de manutenção dos equipamentos e jardins é precária. Mesmo assim, são muitos os pontos positivos, tais como: o frequente uso do local, diversidade de faixas etárias dos usuários, presença de grupos de pessoas interagindo, uso de todos os espaços oferecidos, e existência de 


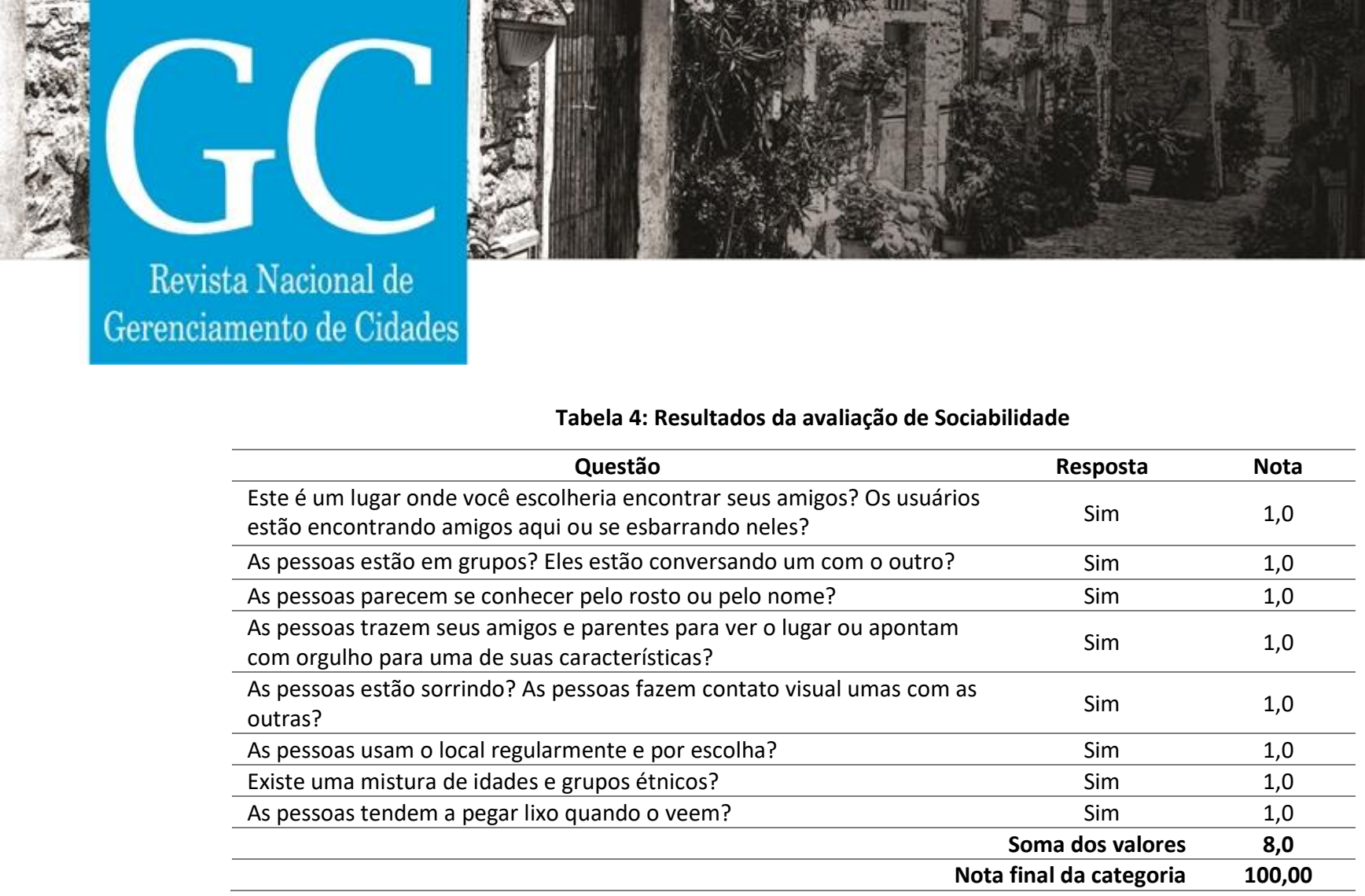

Fonte: elaborado pelos autores, 2019.

O desempenho como um todo da praça foi positivo e satisfatório, a categoria que teve a nota mais baixa foi conforto e imagem com 71,43 , mas ainda assim sua classificação foi boa. Por outro lado, a sociabilidade teve pontuação perfeita de 100. A nota final de qualidade e vitalidade (sucesso do espaço) foi 86,16, desta forma a Praça Luiz Zuiani é classificada como ótima e bemsucedida.

Essa praça já foi avaliada anteriormente por Fontes et al. (2005), os principais problemas encontrados na época foram a presença de equipamentos danificados como bancos e o busto do Luiz Zuiani e falta de paisagismo adequado. A falta de manutenção apropriada ainda é o ponto de maior problema do espaço, apesar de ter passado por uma recuperação em 2010 (Prefeitura Municipal de Bauru, 2010), quando o busto foi recuperado e foi inserida a academia ao ar livre. A construção da academia e de um edifício residencial no seu entorno podem ter colaborado para uma maior apropriação do espaço, pois em seu estudo, Fontes et al. (2005), descreve a praça com função de passagem e pouca permanência. Em contraponto, a presente pesquisa evidenciou que o espaço possui características recreativas e de permanência, além de passagem. O que comprova a importância do entorno residencial e da presença de equipamentos e atividades para a apropriação de espaços públicos, como mencionado no próprio artigo.

\section{CONCLUSÃO}

A aplicação do método proposto pelo PPS na avaliação na Praça Luiz Zuiani, em Bauru-SP, ressaltou a sua eficiência na identificação dos aspectos que contribuem para a qualidade local e que influenciam a sua vitalidade e, consequentemente, o sucesso desse importante espaço recreativo. Por outro lado, os aspectos negativos encontrados indicam fragilidades locais, que comprometem a sua vitalidade e que devem ser revistos pelos gestores urbanos. 
Dessa forma, os resultados obtidos constituem importantes subsídios para intervenções projetuais no local, assim como para o projeto de novos espaços públicos de permanência. Além disso, a aplicação do método em diferentes espaços públicos permite a comparação entre eles e pode constituir em um importante instrumento de gestão para auxiliar intervenções visando a qualidade local.

\section{REFERÊNCIAS BIBLIOGRÁFICAS}

ANGELIS, Bruno Luiz Domingos de; NETO, Generoso de Angelis. Os Elementos de Desenho Das Praças de MaringáPR. Acta Scientiarum: Technology, v. 22, n. 0, p. 1445-1454, 1 maio 2000.

FERNANDES, Ana Cristina Teixeira Dias. Metodologias de Avaliação da Qualidade dos Espaços Públicos. 2012. Dissertação (mestrado) - Faculdade de Engenharia da Universidade do Porto, Porto, Portugal, 2012.

FONTES, Maria Solange Gurgel de Castro et al. Qualidade dos principais espaços públicos de Bauru-SP. In: VIII Encontro Nacional e IV Encontro Latino-Americano sobre Conforto no Ambiente Construído, 2005, Maceió. Anais... Maceió: ANTAC, 2005. v. 1, p.697-705.

GEHL, Jan. Cidades para pessoas. 2. ed. São Paulo: Perspectiva, 2013.

IBGE. Instituto Brasileiro de Geografia e Estatística. Brasil, SP, Bauru, Panorama. Cidades.ibge.gov, 2017. Disponível em: <https://cidades.ibge.gov.br/brasil/sp/bauru/panorama>. Acesso em: 24 out. 2018.

KOSSAK-JAGOCZINSKA, Anna. Presentation of functional, PPS and hall's methods in comparison with other research on space quality. Civil and Environmental Engineering Reports, p. 83-93, 2015.

MARCUS, Clare Cooper; FRANCIS, Carolyn. People Places: design guidelines for urban open space. 2. ed. [s.l.] John Wiley \& Sons, Inc., 1998.

PPS. Project for public spaces. What makes a successful place? Pps.org, c2018. Disponível em:

<https://www.pps.org/article/grplacefeat>. Acesso em 20 fev. 2019.

Prefeitura Municipal de Bauru, Praça Luiz Zuiani está pronta para receber a quinta Academia ao Ar Livre, 2010.

Disponível em:<http://www.bauru.sp.gov.br/Materia.aspx?n=341>. Acesso em: 08 maio 2019.

ROBBA, Fabio; MACEDO, Silvio Soares. Praças brasileiras. 3. ed. São Paulo: EDUSP, 2010.

ROMERO, Marta Adriana Bustos. Arquitetura bioclimática do espaço público. 3. ed. [s.I.] UnB, 2001.

SAKIP, Siti Rasidah Md; AKHIR, Norizan Mt; OMAR, Siti Syamimi. Determinant Factors of Successful Public Parks in Malaysia. Asian Conference on Environment-Behaviour Studies, Chung-Ang University, Seul, Coreia do Sul, 2014.

SCUDO, Gianni; DESSI, Valentina. Thermal comfort in urban space renewal. In: The 23 Conference on Passive and Low Energy Architecture, 2006, Geneva Anais... Geneva, 2006.

VOORDT, Theo J. M. Van Der; WEGEN, Herman B. R. Van. Arquitetura sob o olhar do usuário. São Paulo: Oficina de Textos, 2013. 